\title{
SPFI: Shape-Preserving Choquet Fuzzy Integral for Non-Normal Fuzzy Set-Valued Evidence
}

\author{
Timothy C. Havens, Anthony J. Pinar \\ Department of Electrical and \\ Computer Engineering \\ Department of Computer Science \\ Michigan Technological University \\ Houghton, MI 49931, USA \\ e-mail: \{thavens, ajpinar\}@mtu.edu
}

\author{
Derek T. Anderson \\ Department of Electrical Engineering \\ and Computer Science \\ University of Missouri \\ Columbia, MO, 65201, USA \\ e-mail: andersondt@missouri.edu
}

\author{
Christian Wagner \\ Department of Computer Science \\ University of Nottingham \\ Nottingham, NG8 1BB, UK \\ e-mail: Christian.Wagner@nottingham.ac.uk
}

\begin{abstract}
Information or data aggregation is an important part of nearly all analysis problems as summarizing inputs from multiple sources is a ubiquitous goal. In this paper we propose a method for non-linear aggregation of data inputs that take the form of non-normal fuzzy sets. The proposed shape-preserving fuzzy integral (SPFI) is designed to overcome a well-known weakness of the previously-proposed sub-normal fuzzy integral (SuFI). The weakness of SuFI is that the output is constrained to have maximum membership equal to the minimum of the maximum memberships of the inputs; hence, if one input has a small height, then the output is constrained to that height. The proposed SPFI does not suffer from this weakness and, furthermore, preserves in the output the shape of the input sets. That is, the output looks like the inputs. The SPFI method is based on the well-known Choquet fuzzy integral with respect to a capacity measure, i.e., fuzzy measure. We demonstrate SPFI on synthetic and real-world data, comparing it to the SuFI and non-direct fuzzy integral (NDFI).

Keywords-Fuzzy Integral; Choquet Integral; Capacity Measure; Fuzzy Measure; Aggregation; Fusion
\end{abstract}

\section{INTRODUCTION}

Information aggregation is a basic operation in several areas: machine learning, signal processing, distributed sensing, robotics, crowd-sourcing, etc. In this paper, we address a type of aggregation that has been studied in several previous works [1-6]: the Choquet fuzzy integral (CFI) with respect to a capacity measure (CM) - more popularly called a fuzzy measure, at least in this venue. The combination of the CFI and $\mathrm{CM}$ produces a non-linear aggregation method that is essentially a compressed parameterization of a set of linear convex sums (LCS), one for each possible ranking order of the inputs. Whereas there are $n$ ! possible rank orderings of $n$ inputs, the CFI/CM parameterizes the associated $n$ ! LCSs using $\left(2^{n}-2\right)$ free parameters. To illustrate the efficiency of the CFI/CM aggregation as compared to that of $n$ ! LCSs, we show the compression ratio in Fig. 1. At only 10 sources, there are already $>10^{4} \times$ more terms in $n$ ! LCSs than that of the $\left(2^{n}-2\right)$ terms in the CFI/CM. Section I-A describes CMs in more detail and Section I-B then details how the CFI can be used to aggregate inputs with respect to a CM.

While numerous applications of the conventional CFI/CM have been written about, this paper specifically addresses the

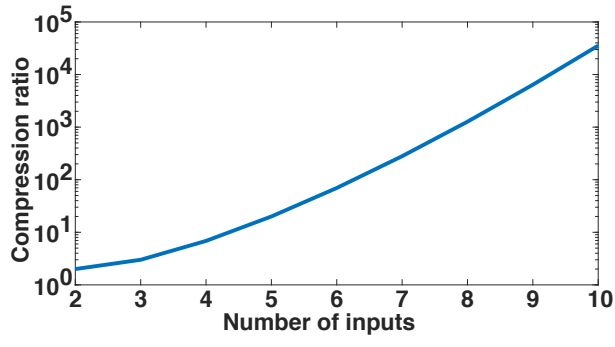

Fig. 1: Compression ratio $(n \cdot n !):\left(2^{n}-2\right)$ of the number of terms in all possible rankings of $n$ sources compared to the number of free parameters in the CFI/CM.

extension of CFI/CM aggregation to inputs that take the form of fuzzy sets (FS). Much has been written on how to extract intervals and FSs from data in real-world problems [7, 8], and numerous CFI extensions to address FS inputs have been proposed [6, 9-13]. An unsolved problem in this regard is non-normal FS inputs, or inputs that do not have a heighti.e., maximum membership-equal to 1 . One extension in particular that addresses non-normal FS inputs is the subnormal fuzzy integral (SuFI). But SuFI is plagued by a major weakness, the output FS has a maximum membership value equal to the minimum of the maximum memberships of all inputs. That is, the "shortest" input drives the maximum membership of the output. Figure 2 illustrates this problem for an extreme example of aggregating two FSs, $A$ and $B$. Set $A$ has a maximum membership of 0.1 ; even if set $A$ is given zero weight in the aggregation, the SuFI result is limited to a height of 0.1 , producing the orange membership function labeled $C_{g}(A, B)$. Intuitively, the aggregation of these two sets if $A$ has zero weight should be exactly $B$. Hence, we propose in this paper a shape-preserving fuzzy integral (SPFI) that addresses the weakness of SuFI.

Section II introduces the proposed SPFI. Some synthetic and real-world examples are presented in Section III and then we conclude. We now continue by presenting a brief introduction to CMs and CFIs, formally presenting the notation used throughout this paper. 


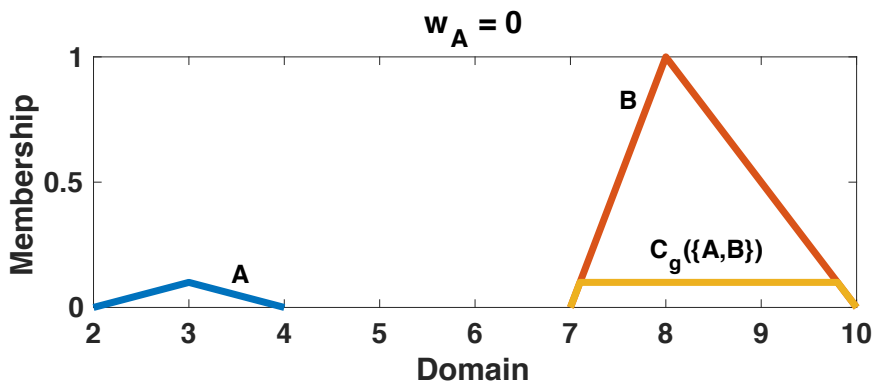

Fig. 2: Example of a two-input fuzzy integral where the weight on set $A$ is zero. This illustrates how $B$ is cut-off where alphacuts of $A$ do not exist.

\section{A. Capacity measures}

A measurable space is the tuple $(X, \Omega)$, where $X$ is a set and $\Omega$ is a $\sigma$-algebra or set of subsets of $X$ such that

P1. $X \in \Omega$;

P2. For $A \subseteq X$, if $A \in \Omega$, then $A^{c} \in \Omega$;

P3. If $A_{i} \in \Omega$, then $\bigcup_{i=1}^{\infty} A_{i} \in \Omega$.

A $\mathrm{CM}$ is a set-valued function, $g: \Omega \rightarrow[0,1]$, with the following properties:

P4. (Boundary conditions) $g(\emptyset)=0$ and $g(X)=1$;

P5. (Monotonicity) If $A, B \in \Omega$ and $A \subseteq B, g(A) \leq g(B)$.

If $\Omega$ is an infinite set, then there is also a third property guaranteeing continuity; in practice and in this paper, $\Omega$ is finite and thus this property is unnecessary. The CM values of the singletons, $g\left(\left\{x_{i}\right\}\right)=g^{i}$ are commonly called the densities.

Arguably, the most popular CM, i.e., fuzzy measure, is the Sugeno $\lambda$-measure, which has the attractive property of being able to be defined completely by the values of the densities. The $\lambda$-measure has the following additional property. For $A, B \in \Omega$ and $A \cap B=\emptyset$,

$$
g_{\lambda}(A \cup B)=g_{\lambda}(A)+g_{\lambda}(B)+\lambda g_{\lambda}(A) g_{\lambda}(B),
$$

where it can be shown that $\lambda$ can be found by solving [14]

$$
\lambda+1=\prod_{i=1}^{n}\left(1+\lambda g^{i}\right), \quad \lambda>-1 .
$$

More recent works on CMs have proposed learning CMs from training data [15].

\section{B. Choquet integrals}

There are many forms of the fuzzy integral; see [14] for detailed discussion. In practice, FIs are mostly used for evidence fusion [1-6]. They combine sources of information by accounting for both the support of the question (the evidence) and the expected worth of each subset of sources (as supplied by the CM $g$ ). Here, we focus on the fuzzy Choquet integral (CFI), proposed by Murofushi and Sugeno [16, 17]. Let $h: X \rightarrow \mathcal{R}$ be a real-valued function that represents the evidence or support of a particular hypothesis. ${ }^{1}$ The discrete (finite $\Omega$ ) Choquet integral is defined as

$$
\begin{aligned}
\int_{C} h \circ g & =C_{g}(h)=\sum_{i=1}^{n} h\left(x_{\pi(i)}\right)\left[g\left(\Pi_{i}\right)-g\left(\Pi_{i-1}\right)\right], \\
& =\mathbf{w}_{\pi}^{T} \mathbf{h}_{\pi},
\end{aligned}
$$

where $\pi$ is a permutation of $X$, such that $h\left(x_{\pi(1)}\right) \geq$ $h\left(x_{\pi(2)}\right) \geq \ldots \geq h\left(x_{\pi(n)}\right), \Pi_{i}=\left\{x_{\pi(1)}, \ldots, x_{\pi(i)}\right\}$, and $g\left(A_{0}\right)=0[11,18]$. At $(2 \mathrm{~b})$, we have simply reformulated (2a) as the dot-product of the vectors $\mathbf{w}_{\pi}$ and $\mathbf{h}_{\pi}$, where

$$
\begin{aligned}
\mathbf{h}_{\pi} & =\left(h\left(x_{\pi(1)}\right), h\left(x_{\pi(2)}\right), \ldots, h\left(x_{\pi(n)}\right)\right)^{T}, \\
\mathbf{w}_{\pi} & \left.=\left(g\left(\Pi_{1}\right),\left(g\left(\Pi_{2}\right)-g\left(\Pi_{1}\right)\right), \ldots,\left(1-g_{(} \Pi_{n-1}\right)\right)\right)^{T} .
\end{aligned}
$$

The insight of (2b) is that the CFI with respect to the CM is a collection of $n$ ! linear-order statistics on $h$, one for each possible sort order of the evidence $h$. The elements of $\mathbf{w}_{\pi}$ are simply the weights of each evidence value, as represented by the gain in the CM up through the lattice. More details of the properties of CFIs and fuzzy integrals in general can be found in $[11,18,19]$.

Example 1. As a simple example, let us consider three sources, $A, B$, and $C$, each providing respective evidence, $h_{A}$, $h_{B}$, and $h_{C}$. The CM for these sources is shown in Fig. 3(b), e.g., $g(\{A\})=0.1$ and $g(\{A, B\})=0.95$. If $h_{A}=0.1$, $h_{B}=0.01$, and $h_{C}=0.9$, then the CFI at (2) is

$$
\begin{aligned}
C_{g}(h)= & h_{C} g(\{C\})+h_{A}(g(\{A, C\})-g(\{C\})) \\
& +h_{B}(1-g(\{A, C\})), \\
= & 0.9 * 0.8+0.1 *(0.9-0.8)+0.01 *(1-0.9), \\
= & 0.731 .
\end{aligned}
$$

In this example, $\mathbf{w}_{\pi}=(0.8,0.1,0.1)^{T}$ and $\mathbf{h}_{\pi}=$ $(0.9,0.1,0.01)^{T}$.

In some cases, the evidence $h$ cannot, or should not, be represented simply by numbers; $h$ would be better represented as an interval-valued or FN-valued function-or, as we will argue later, by a general FS. An example is the survey question, "How many bottles of wine should I purchase for the reception?" Many people would answer this question with an interval, e.g., "between 20 and 30," or a FN, e.g., "about 25." Furthermore, there is much work on how to extract intervals and fuzzy sets from data in real-world problems $[7,8]$. Extensions of the fuzzy CFI have been proposed for both interval-valued, fuzzy number-valued, and normal nonconvex integrands $[6,9-13]$, which we now discuss.

Let $I(\mathcal{R})=\left\{\bar{u} \subset \mathcal{R} \mid \bar{u}=\left[u^{-}, u^{+}\right], u^{-} \leq u^{+}\right\}$be the set of all closed intervals over the real numbers. Dubois and Prade [20] showed that if a function $\phi$ is continuous and nondecreasing, then, when defined on intervals, $\phi$ produces an interval, the endpoints of which are equal to the function values on the lower and upper bound of the individual intervals,

\footnotetext{
${ }^{1}$ Generally, when dealing with information fusion problems it is convenient to have $h: X \rightarrow[0,1]$, where each source is normalized to the unit-interval.
} 
viz., $\phi(\bar{u})=\left[\phi\left(u^{-}\right), \phi\left(u^{+}\right)\right]$. This approach benefits us in computing CFIs for intervals as they are continuous and nondecreasing. Let $\bar{h}: X \rightarrow I(\mathcal{R})$, where $\bar{h}_{i}=\bar{h}\left(x_{i}\right)=\left[h_{i}^{-}, h_{i}^{+}\right]$ is the interval-valued integrand (evidence). The CFI on $h$ is

$$
\int \bar{h} \circ g=\left[\int h^{-} \circ g, \int h^{+} \circ g\right],
$$

where the output $\int \bar{h} \circ g$ is itself interval-valued.

Now, let $\hat{H}: X \rightarrow F S(\mathcal{R})$ be a non-convex, normal FSvalued integrand. ${ }^{2}$ Hence, the height of each FS in $\hat{H}$ is 1 . Using the Extension Principle [21], the CFI of $\hat{H}$ w.r.t. $g$ is then defined as

$$
\left(\int \hat{H} \circ g\right)(a)=\sup _{\alpha \in[0,1]}\left\{a \in \int{ }^{\alpha} \hat{H} \circ g\right\},
$$

where ${ }^{\alpha} \hat{H}=\left[{ }^{\alpha} h^{-},{ }^{\alpha} h^{+}\right]$are the closed intervals of the level-cuts of the members of $\hat{H}$ at $\alpha$. Note that ${ }^{\alpha} \hat{H}$ is not necessarily one continuous interval, but a set of intervals (or a discontinuous interval) formed by taking the level-cut of each $\hat{H}_{i} \in \hat{H}$ [22]. Hence ${ }^{\alpha} \hat{H}=$ $\left\{\left[\left({ }^{\alpha} \hat{H}_{1}\right)^{-},\left({ }^{\alpha} \hat{H}_{1}\right)^{+}\right], \ldots,\left[\left({ }^{\alpha} \hat{H}_{n}\right)^{-},\left({ }^{\alpha} \hat{H}_{n}\right)^{+}\right]\right\}$and ${ }^{\alpha} h^{-}=$ $\left\{\left({ }^{\alpha} \hat{H}^{1}\right)^{-}, \ldots,\left({ }^{\alpha} \hat{H}^{n}\right)^{-}\right\}$(and similarly defined for ${ }^{\alpha} h^{+}$). Equation (5) can thus alternatively be written as

$$
\begin{aligned}
& \left(\int \hat{H} \circ g\right)(a)=\bigcup_{\alpha \in[0,1]} \alpha\left[\left(\int{ }^{\alpha} \hat{H} \circ g\right)(a)\right], \\
& =\bigcup_{\alpha \in[0,1]} \alpha\left[\left(\int{ }^{\alpha} h^{-} \circ g\right)(a),\left(\int{ }^{\alpha} h^{+} \circ g\right)(a)\right],
\end{aligned}
$$

where $a$ is the independent variable of the CFI; $a \in \mathcal{R}$ for the CFI.

The problem with the CFI at (5) is that it is undefined for sub-normal FS inputs, that is, there are values of $\alpha$ for which $\alpha$-cuts do not exist in all the inputs. To combat this problem, we previously proposed extensions to the FIs for sub-normal FSs, called SuFI and NDFI [5, 6, 13].

Let $H: X \rightarrow F S(\mathcal{R})$ be a general FS-valued integrand. The sub-normal FI (SuFI), defined as

$$
\begin{aligned}
\left(\int H \circ g\right)(a) & =\sup _{\alpha \in[0, \beta]}\left\{a \in \int{ }^{\alpha} H \circ g\right\}, \\
\beta & =\bigwedge_{i=1}^{n} \operatorname{Height}\left(H_{i}\right),
\end{aligned}
$$

simply computes (5) up to the minimum height of any input FS and then stops. Figures 2 and 6 illustrate SuFI's weakness; it does not maintain the shape of the inputs and one low membership input can severely impact the aggregation result (even if that input has zero-weight in the aggregation).

Hence, we proposed the non-direct FI (NDFI), which instead computes FI aggregation on the (vertical) membership values, rather than the (horizontal) alpha-cuts.

\footnotetext{
${ }^{2}$ Note that this includes fuzzy numbers, as well.
}

\section{Shape-Preserving FuZzy Integral}

Let $\beta_{i}=\operatorname{Height}\left(H_{i}\right)$ indicate the height of FS input $H_{i}$. Then, the shape-preserving FI (SPFI) is calculated by

$$
\left(\int H \circ g\right)(a)=b \sup _{\alpha \in[0,1]}\left\{a \in \int{ }^{\alpha} \operatorname{Norm}(H) \circ g\right\},
$$

where $\operatorname{Norm}(H)=\left\{\operatorname{Norm}\left(H_{1}\right), \ldots, \operatorname{Norm}\left(H_{n}\right)\right\}$ is the set of normalized evidence in $H$. The normalizing function acts upon the membership function of $H_{i}$ as

$$
\operatorname{Norm}\left(H_{i}\right)(x)=H_{i}(x) / \operatorname{Height}\left(H_{i}\right),
$$

which simply scales $H_{i}$ to a height of 1 , making it a normal FS. Hence, the calculation to the right of the equals sign at (8) is simply a FI of FNs. The scaling factor $b$ at (8) is the height of the result, calculated as

$$
\begin{aligned}
b & =\sum_{i=1}^{n} \operatorname{Height}\left(H_{\theta(i)}\right)\left[g\left(\Theta_{i}\right)-g\left(\Theta_{i-1}\right)\right], \\
& =\mathbf{w}_{\theta}^{T}\left(\operatorname{Height}\left(H_{\theta}\right)\right),
\end{aligned}
$$

where $\theta(i)$ is a sorting function of $H$ (nominally, from greatest to least) and $\Theta_{i}=\left\{H_{\theta(1)}, \ldots, H_{\theta(i)}\right\}$. Essentially, the height $b$ is the result of a FI of the heights of the evidence, where the sorting is determined by a relation on $H$. This begs the question, how is this sorting done?

In the conventional fuzzy integral at (2a), the sorting is done such that $h\left(x_{\pi(1)}\right) \geq h\left(x_{\pi(2)}\right) \geq \ldots \geq h\left(x_{\pi(n)}\right)$. The sorting function $\theta$ in (10a) similarly seeks to sort the inputs $H$ from greatest to least. While there are numerous works on ordering FSs, in this work we order based on the center of mass of the core of each normalized FS. Let $C=\left\{c_{1}, \ldots, c_{n}\right\}$ be these centers-of-mass, where $c_{1}$ is the center-of-mass of the core of $\operatorname{Norm}\left(H_{1}\right)$, etc. Then, $\theta$ sorts so that $c_{\theta(1)} \geq c_{\theta(2)} \geq \ldots \geq$ $c_{\theta(n)}$. In the sequel to this work, we will explore other possible orderings of $H$, but the proposed center-of-mass approach is simple to implement and, as you will see, it works well in practice.

Example 2. Consider the three FSs $\{A, B, C\}$ in Fig. 3(a) as input evidence $H$ to the SPFI. The CM $g$ for this example is shown in Fig. 3(b).

The first step of SPFI is to normalize the evidence, as shown in Fig. 3(c), and then perform the CFI at (5) on these normalized sets, the result of which is labeled as $\mathrm{SPFI}_{\text {norm }}$. Finally, the height of the final result is calculated by the FI on the heights of the input evidence. The respective heights of $A, B$, and $C$ are $1,0.8$, and 0.5 and the respective core centers-of-mass are $c_{A}=1, c_{B}=3$ and $c_{C}=9$. Hence, the height calculation at (10a) with respect to the CM shown in view (b) is

$$
b=0.5 * 0.8+0.8 *(0.85-0.8)+1 *(1-0.85)=0.59,
$$

where, equivalently, if one wished to use the vector calculation at $(10 \mathrm{~b})$, then $\mathbf{w}_{\theta}=(0.8,0.05,0.15)^{T}$ and $\operatorname{Height}\left(H_{\theta}\right)=$ $(0.5,0.8,1)^{T}$. The final result of the SPFI is shown in Fig. 3(a). This example shows that the SPFI is able to preserve 


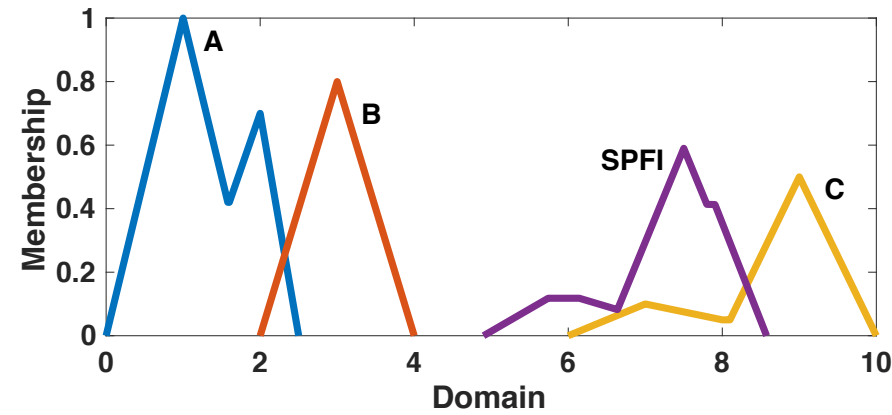

(a) Input $\hat{H}$ and SPFI result

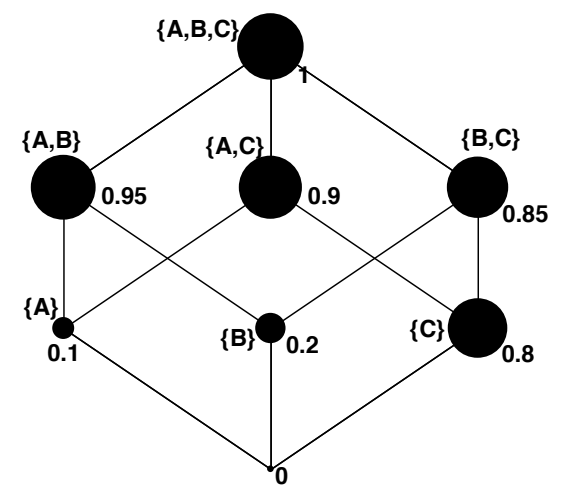

(b) $\mathrm{CM} g$ lattice

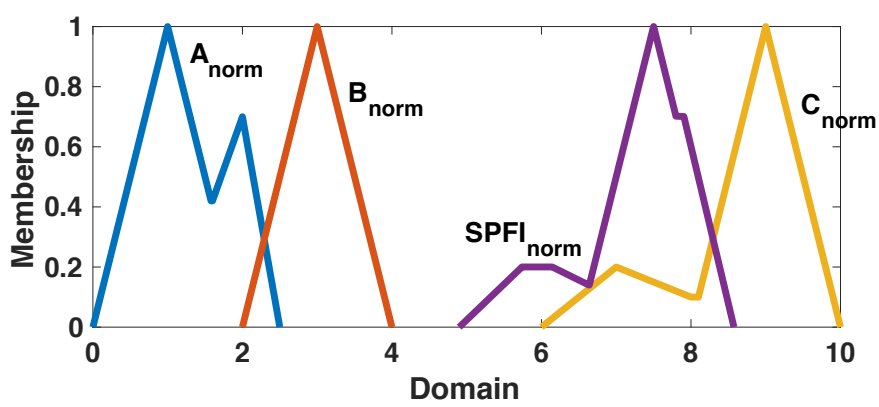

(c) Normalized $\hat{H}$

Fig. 3: Example 1 illustrating the SPFI on three input sets.

the aggregated shape of the three FS inputs, while giving what we consider to be a reasonable answer, say "a medium membership in a little less than 8."

We now contrast the SPFI example with the result that would be obtained with SuFI and NDFI. Figure 4 illustrates these results. As we previously showed, SuFI is unable to produce an output at memberships greater than the lowest height evidence $H$; here, that is 0.5 . While, for this example, SuFI and SPFI results are not that different, if we adjust the $\mathrm{CM}$ to put more weight on inputs $A$ and $B$, we see significant difference-see Fig. 5. As this figure shows, the SuFI result is cut-off at a height of 0.5. The SPFI result successfully aggregates the three sets, maintaining the shape of the inputs while giving a result that accurately depicts that the $\mathrm{CM}$ is putting higher weight on inputs $A$ and $B$.

The NDFI result for Example 2 is significantly different

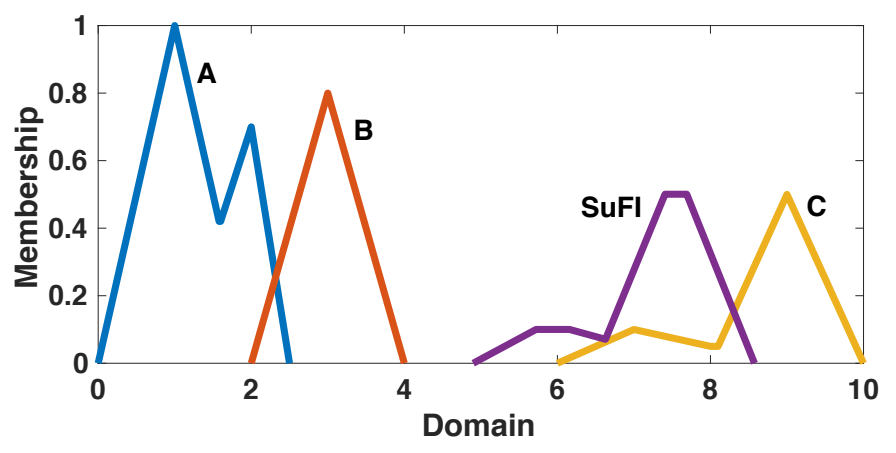

(a) $\mathrm{SuFI}$

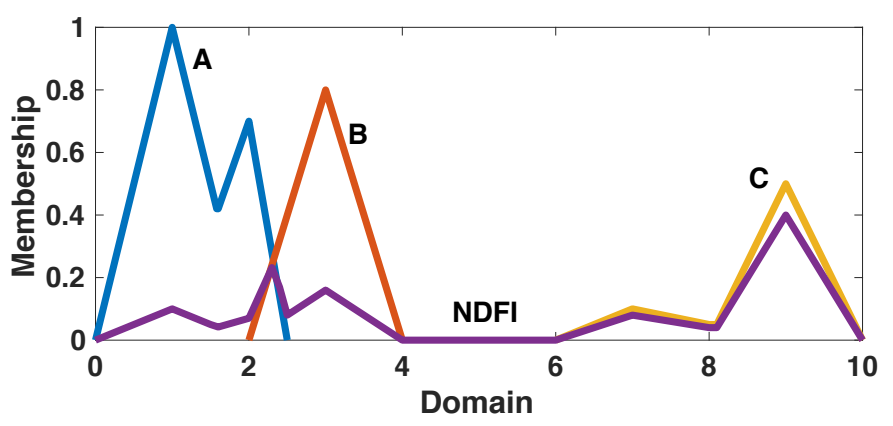

(b) NDFI

Fig. 4: Results of SuFI and NDFI with Example 2 inputs and fuzzy measure.

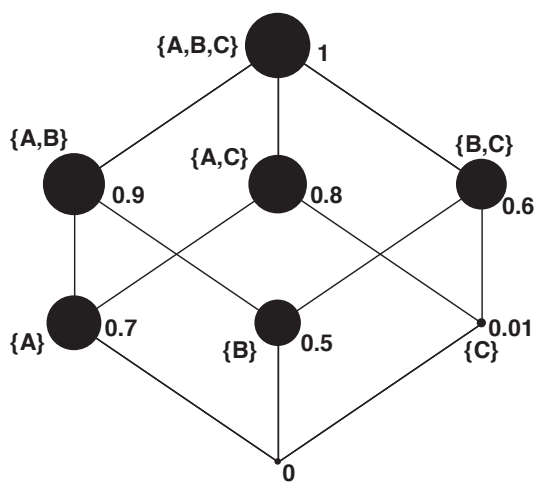

(a) $\mathrm{CM} g$ lattice

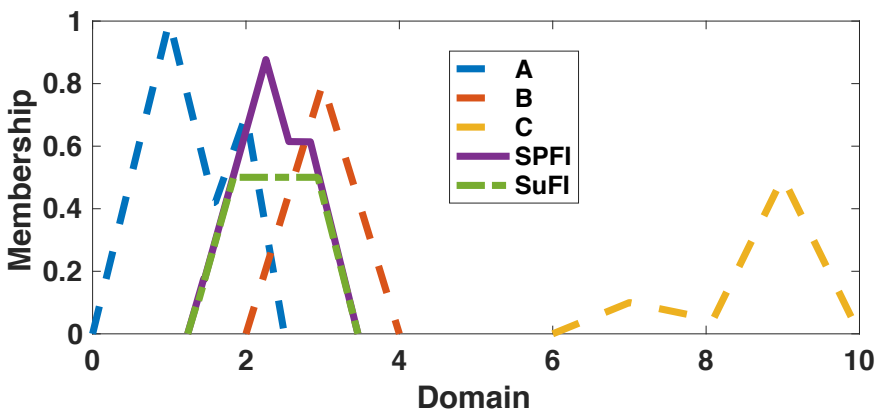

(b) SuFI vs. SPFI

Fig. 5: Results of SuFI and SPFI with Example 2 inputs and capacity measure that puts more weight on sources $A$ and $B$. 

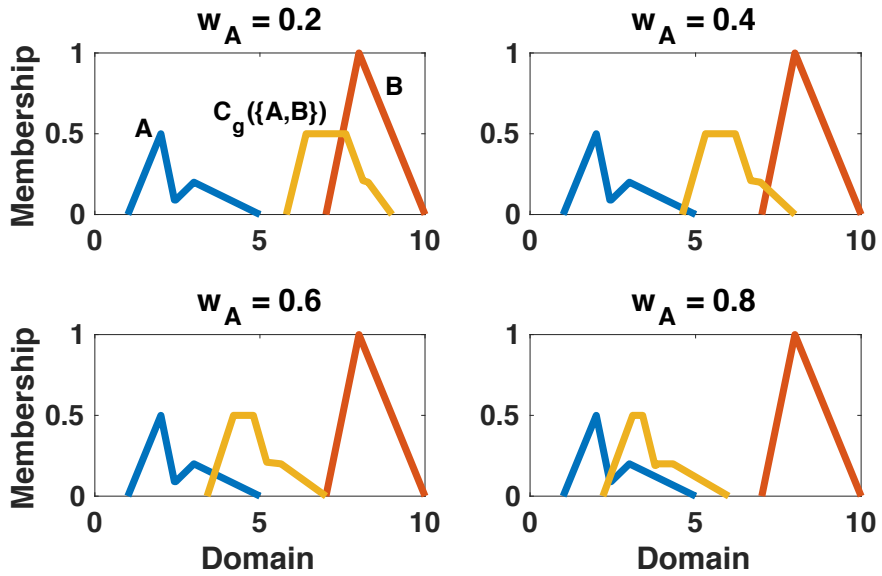

Fig. 6: Demonstration of a two-input SuFI where the weight on set $A$ is incremented from 0.2 to 0.8 . While not as drastic as in Fig. 2, this shows how the aggregation is cut-off above the height of $A$, i.e., $\alpha>0.5$.

than the SuFI and SPFI results. This is because the NDFI is fundamentally a different type of aggregation. The NDFI result shows strong membership in $C$, as is represented in the CM; however, there is also some membership shown down in the region of $A$ and $B$. We hesitate to say that the NDFI is wrong, as we have shown its utility in previous works [5] and will show that it has its strength for a certain type of problem later, but we argue that the SPFI result is the most interpretable of the three algorithms.

\section{EXPERIMENTS}

\section{A. Synthetic Example}

Consider the two FS inputs, $A$ (blue) and $B$ (red), shown in Figs. 6 and 7. In this example, we show a simple aggregation between these two sets with the intention of illustrating how the SPFI better preserves the shape of the high weight inputs. In this example, we adjust the CM as to put more (or less) weight on each set. Hence, the output should look more like the set that has the highest weight. Figure 6 shows the output of the SuFI algorithm. As this illustration shows, even if the weight on $A$ is 0.2 , and thus $w_{B}=0.8$, the output FS is cutoff at the height of $A$. Furthermore, the output doesn't even look like $B$.

Let us now examine the behavior of the SPFI algorithm, shown in Fig. 7. In this case, when the weight on $A$ is large (as shown in the lower right of the figure), the output set looks like set $A$, whereas if the weight on $A$ is small (as shown in the upper left of the figure), the output set looks like $B$. This illustration clearly shows the strength of the SPFI aggregation. We now turn to an example with real-world data.

\section{B. Age at death estimation}

Consider the real-world example, first presented in [5], in forensic anthropology of estimating the age-at-death of human skeletal remains. Table I shows eight aging methods, each providing an interval estimation of age-at-death of skeletal
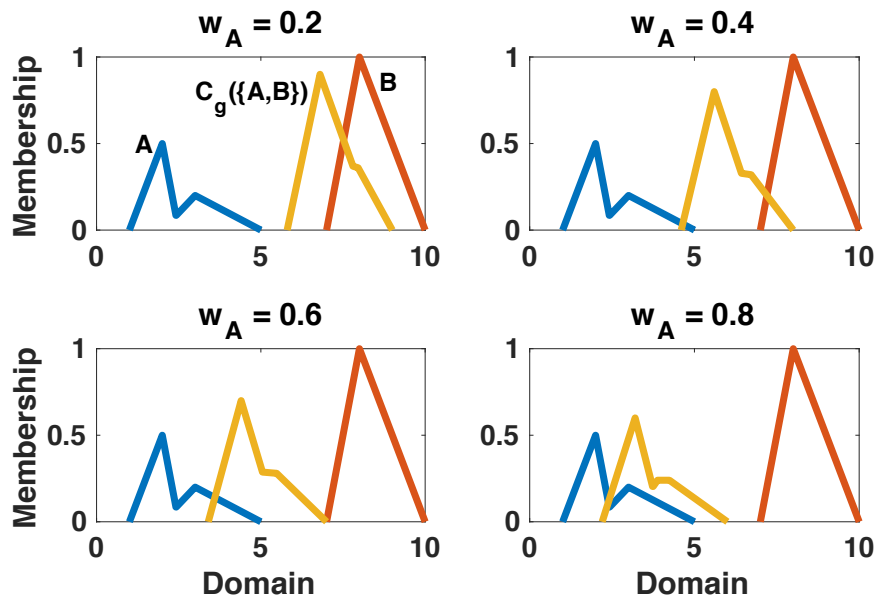

Fig. 7: Demonstration of SPFI for the same sets shown in Fig. 6. This shows that our proposed method maintains a more preferred aggregate shape of the two inputs.

TABLE I: Input for Age-at-Death Example [5, 6]

\begin{tabular}{r|c|c|c}
\hline \hline Aging Method & Quality & Est. Age & Reliability \\
\hline Pubic Symphysis (PS) & 0.6 & $35-39$ & 0.57 \\
Auricular Surface (AS) & 0.8 & $35-39$ & 0.72 \\
Ectocranial Sutures-vault (ESv) & 0.2 & $25-74$ & 0.59 \\
Ectocranial Sutures-lateral (ESl) & 0.5 & $23-63$ & 0.59 \\
Sternal Rib Ends (SRE) & 0.5 & $33-42$ & 0.75 \\
Endocranial Sutures (ES) & 0.4 & $35-39$ & 0.51 \\
Proximal Humerus (PH) & 0.3 & $37-86$ & 0.44 \\
Proximal Femur (PF) & 0.7 & $25-76$ & 0.56 \\
\hline
\end{tabular}

remains. The bones used in each of these methods is assigned a Quality by the forensic anthropologist between 0 and 1, describing the condition of the sample. The Reliability of each test is shown in the last column; this reliability score is used to populate the densities of the FM $g^{i}$. The remaining entries of the FM are computed by the Sugeno $\lambda$-measure.

Each aging method is mapped to an interval FS, where the interval is the estimated age-at-death and the height is the quality of the measurement, shown in Fig. 8(a). So, the width of the interval indicates the uncertainty in the measurement, while the height indicates the membership of that measurement in the set of 'estimated age-at-death' as given by the quality of the bone sample. These intervals are then fused to produce an aggregate FS of the membership of each age at death.

Figure 8(b) shows the output of the three FI-based aggregations. The NDFI is our previously proposed algorithm, which was built specifically for the age-at-death problem. As we have stated before, the strength of the NDFI approach is that it shows a clear high membership around 35 years old, while also showing the lower confidence regions surrounding that more certain estimate. The SPFI and the SuFI both indicate an interval of 35 to 72 years, the only difference being the membership over that interval. The membership of the SuFI is driven simply by the lowest membership interval in the set of inputs, i.e., the ESv aging method. The SPFI membership is truly an aggregate of all the memberships of the interval inputs according to the weights derived by the reliability scores and 


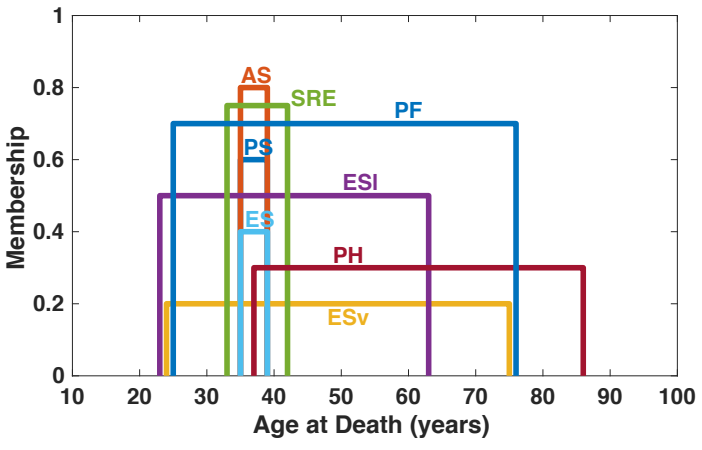

(a) Input Sets

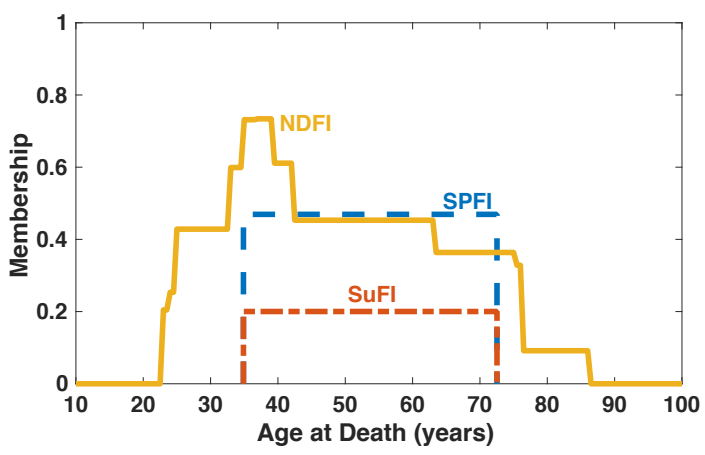

(b) Output Sets

Fig. 8: Example showing estimation of skeleton age-at-death. Aging methods shown in Table I.

\section{subsequent FM.}

For this specific problem, age at death estimation, we believe that the NDFI is the preferred tool. First, the NDFI was specifically designed for this problem. Second, a result of the first point, the NDFI shows nicely the areas in which the aging methods agree and how much they agree (weighted by the reliability score). In contrast, the SPFI and SuFI simply return interval-based sets that don't provide a more detailed view of the regions where the methods agree and disagree. We do not see this as a weakness of the SPFI, but simply as a result of how the SPFI was designed-as an aggregation scheme which produces output that preserve the shapes of the inputs. For the specific age at death estimation problem, the NDFI is the preferred aggregation scheme.

\section{CONCLUSION}

This paper proposed a new method, called SPFI, for nonlinear aggregation of non-normal fuzzy sets with the intention of having the output of the aggregation preserve the shape of the inputs. We showed through synthetic examples that this method addresses a major weakness in the previously leading algorithm, SuFI.

Future work in this area will focus on further developing this method for real-world problems and proposing an efficient way for representation and aggregation of non-convex fuzzy sets (convex fuzzy sets are computationally efficient to represent by simply storing the intervals at each alpha-cut).

\section{ACKNOWLEDGEMENT}

Superior, a high-performance computing infrastructure at Michigan Technological University, was used in obtaining results presented in this publication.

\section{REFERENCES}

[1] M. Grabisch, Fuzzy Measures and Integrals: Theory and Applications. New York: Physica-Verlag, 2000, ch. Fuzzy integral for classification and feature extraction, pp. 415-434.

[2] J. M. Keller, P. Gader, and A. K. Hocaoglu, Fuzzy Measures and Integrals: Theory and Applications. New York: Physica-Verlag, 2000, ch. Fuzzy integral in image processing and recognition, pp. 435-466.

[3] S. Auephanwiriyakul, J. M. Keller, and P. Gader, "Generalized Choquet fuzzy integral fusion," Information Fusion, vol. 3, pp. 69-85, 2002.

[4] H. Tahani and J. M. Keller, "Information fusion in computer vision using the fuzzy integral," IEEE Trans. Systems Man Cybernet., vol. 20, no. 3, pp. 733-741, 1990.

[5] M. Anderson, D. T. Anderson, and D. Wescott, "Estimation of adult skeletal age-at-death using the Sugeno fuzzy integral," American Journal of Physical Anthropology, vol. 142, no. 1, pp. 30-41, 2009.

[6] D. T. Anderson, T. C. Havens, C. Wagner, J. M. Keller, M. F. Anderson, and D. J. Wescott, "Extension of the fuzzy integral for general fuzzy set-valued information," IEEE Trans. Fuzzy Systems, vol. 22, no. 6, pp. 1625-1639, 2014.

[7] D. Wu, J. M. Mendel, and S. Coupland, "Enhanced interval approach for encoding words into interval type-2 fuzzy sets and its convergence analysis," IEEE Trans. Fuzzy Systems, vol. 20, no. 3, pp. 499-513, 2012.

[8] C. Wagner, S. Miller, J. Garibaldi, D. T. Anderson, and T. C. Havens, "From interval-valued data to general type-2 fuzzy sets," IEEE Trans. Fuzzy Systems, vol. 23, no. 2, 2015.

[9] D. Zhang and Z. Wang, "Fuzzy integrals of fuzzy valued functions," Fuzzy Sets and Systems, vol. 54, pp. 63-67, 1993.

[10] R. Yang, Z. Wang, P. Heng, and K. Leung, "Fuzzified Choquet integral with a fuzzy-valued integrand and its application on temperature prediction," IEEE Trans. SMC-B, vol. 38, no. 2, pp. 367-380, Apr. 2008.

[11] M. Grabisch, H. T. Nguyen, and E. A. Walker, Fundamentals of Uncertainty Calculi, With Applications to Fuzzy Inference. Dordrecht: Kluwer Academic, 1995.

[12] T. C. Havens, D. T. Anderson, and J. M. Keller, "A fuzzy Choquet integral with an interval type-2 fuzzy number-valued integrand," in Proc. IEEE Int. Conf. Fuzzy Systems, Barcelona, Spain, 2010, pp. 1-8.

[13] D. T. Anderson, T. C. Havens, C. Wagner, J. M. Keller, M. Anderson, and D. Wescott, "Sugeno fuzzy integral generalizations for sub-normal fuzzy set-valued inputs," in Proc. IEEE Int. Conf. Fuzzy Systems, Brisbane, Australia, 2012, pp. 1-8.

[14] M. Grabisch, T. Murofushi, and M. Sugeno, Eds., Fuzzy Measures and Integrals: Theory and Applications. New York: Physica-Verlag, 2000.

[15] T. C. Havens, D. T. Anderson, and C. Wagner, "Data-informed fuzzy measures for fuzzy integration of intervals and fuzzy numbers," IEEE Trans. Fuzzy Systems, vol. 23, no. 5, pp. 1861-1875, oct 2015.

[16] G. Choquet, "Theory of capacities," Analles de l'Institit Fourier, vol. 5, pp. 131-295, 1953.

[17] T. Murofushi and M. Sugeno, "An interpretation of fuzzy measure and the Choquet integral as an integral with respect to a fuzzy measure," Fuzzy Sets and Systems, vol. 29, no. 2, pp. 201-227, 1989.

[18] M. Sugeno, "Theory of fuzzy integral and its applications," Ph.D. dissertation, Tokyo Institute of Technology, 1974.

[19] M. Grabisch, "Fuzzy integral in multicriteria decision making," Fuzzy Sets and Systems, vol. 69, pp. 279-298, 1995.

[20] D. Dubois and H. Prade, Fuzzy Sets and Systems: Theory and Application. New York, NY: Academic Press, 1980.

[21] D. Dubois, W. Ostawiewicz, and H. Prade, "Fuzzy sets: history and basic notions," in Fundamentals of Fuzzy Sets, D. Dubois and H. Prade, Eds. Boston, MA: Kluwer, 2000, pp. 21-124.

[22] C. Wagner, D. T. Anderson, and T. C. Havens, "Generalization of the fuzzy integral for discontinuous interval- and non-convex interval fuzzy set-valued inputs," in Proc. Int. Conf. Fuzzy Systems, 2013, pp. 1-8. 